\title{
Research Paper \\ Maintaining and Promoting Techniques for Mental Health Used by Iranian Married Women: A Qualitative Study
}

\author{
*Monir Baradaran Eftekhari ${ }^{1}$, Ameneh Setareh Forouzan², Arash Mirabzadeh ${ }^{3}$, Masoumeh Dejman ${ }^{4}$, Hadi Yousefi ${ }^{5}$
}

\begin{abstract}
1. PhD in Social Determinants of Health (SDH), Assistant Professor, Deputy of Research and Technology, Ministry of Health and Medical Education, Tehran, Iran. 2. Psychiatrist, Associate Professor, Social Determinants of Health Research Center, University of Social Welfare and Rehabilitation Sciences, Tehran, Iran 3. Psychiatrist, Professor, Social Determinants of Health Research Center, University of Social Welfare and Rehabilitation Sciences, Tehran, Iran.

4. PhD in Clinical Neuroscience, Assistant Professor, Social Determinants of Health Research Center, University of Social Welfare and Rehabilitation Sciences, Tehran, Iran. 5. MSc., Department of IT, Faculty of Computer Engineering, University of Tehran, Tehran, Iran.
\end{abstract}

\begin{tabular}{|c|c|}
\hline $\begin{array}{l}\text { Use your device to scan } \\
\text { and read the article online }\end{array}$ & 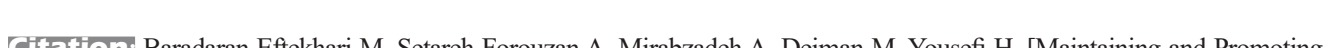 \\
\hline 口대년 & 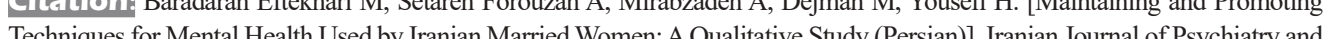 \\
\hline 是4 & Clinical Psychology. 2018; 24(1):70-79. https://doi.org/10.29252/NIRP.IJPCP.24.1.70 \\
\hline 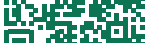 & de:" httralldoi \\
\hline a.ting & dol : https://d01.0rg/10.29252/NIRP.IJPCP.24.1.70 \\
\hline
\end{tabular}

Received: 06 May 2017

Accepted: 01 Nov. 2017

Key words:

Mental health pro-

motion, Women,

Qualitative study

\section{A B STRACT}

Objectives Mental health problems are prevalent especially in women all over the world. These problems have adverse effects on various aspects of life. Interventional programs to mental health promotion related to sociocultural structure and it is needed to know the coping mechanisms and mental health promotion strategies and it is the aim of this study.

Methods This is a qualitative study. The relevant data were collected by in-depth interviews with 15 married women (18-65 years old) in Tehran. Then the obtained data were investigated through content analysis.

Results Married women used four strategies to cope with the stress and promote the mental health status: a) strengthening the spiritual aspects, b) learning the skills of stress management, positive thinking, self-controlling and problem solving, c) seeking help, and d) setting their priorities setting and avoid daily boring tasks.

Conclusion Teaching coping mechanisms based on problem-solving techniques is essential for mental health promotion in married women.

\section{Extended Abstract}

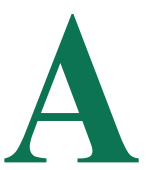

\section{Introduction}

ccording to WHO definition, mental health is a state of wellbeing in which the individuals can recognize their abilities, can cope with the normal stresses of life, work productively and fruitfully, and contribute to their communities. In other words, mental health is beyond the absence of clinical mental illness. In 2004, $1.2 \%$ of deaths in men and $2.2 \%$ of deaths in women were caused by psychological disorders.
These diseases account for one third of years living with disability. In 2004, according to Noorbala et al. survey, using the General Health Questionnaire, $21 \%$ of the Iran total population (25.9\% of women and $14.6 \%$ of men) suffered from mental illness. In 2008, this percentage increased up to $36 \%$ in Tehran (2 to 1 in women than men). Regarding the high prevalence of mental health problems, especially among women and its association with other diseases and complications, the interventional programs should be executed to promote mental health. Different studies indicate that mental health promotion at the community level can reduce social damage. There are several interventional pro-

* Corresponding Author:

Monir Baradaran Eftekhari, PhD

Address: Deputy of Research and Technology, Ministry of Health and Medical Education, Tehran, Iran.

Tel: +98 (21) 81455184

E-mail: mbeftekhari200@gmail.com 
grams in mental health field. Mental health problems are prevalent especially in women all over the world. These problems have adverse effects on various aspects of life. Interventional programs to mental health promotion will be related to sociocultural structure and it is needed to know the different mechanisms and mental health promotion strategies based on married women opinions and it is the aim of this study.

\section{Method}

This is a qualitative study. Data collection started from December 2011 to July 2012. In this study, the participants were selected by purposeful sampling method out of married women, 18 to 65 years old, who were residents in District 22 of Tehran Municipality. The number of interviews was based on data saturation. Each interview lasted 1-1.5 hours, and ended when no new issues pop up. The obtained data were investigated through content analysis.

\section{Results}

Four strategies have been used by married women to promote the mental health status. The strategies are Strengthening the spiritual aspects are strengthening the spiritual aspects, learning the skills of stress management; positive thinking, self-controlling and problem solving; help seeking; and setting the priorities and avoiding daily boring tasks.

According to some participant's opinion, behavioral characteristics like aggression, telling a lie, egoism, jealousy, vanity, irresponsibility contribute in mental health problems. "Jealous people always compare themselves with others and are not at all comfortable". "People who are lying, are always anxious and worried, because they are afraid of revealing their lies". Learning the life skills as stress management, problem solving and so on were other strategies for mental health promotion. Help seeking process consists of "reinforcement of believing in God", support from others and counseling. "When I'm sad, I feel, some supernatural power helps me". Some participants believed that despite advantages of counseling, because of its high cost and social stigma; many people do not seek counseling and it can worsen the already psychological problem. Changing the living condition, such as traveling, entertaining activities, provision of a cheerful environment were the suggested strategies for changing the monotonous rhythm of life and dealing with the problem. Peers are particularly important in this process.

\section{Discussion}

Designing the interventional programs based on married women opinions may be useful to promote mental health in married women. The present study reported and investigated the participant's experiences through 15 in-depth interviews and its results should not be generalized to other conditions for methodological reasons (qualitative approach). All ethical issues (such as conflict of interests, plagiarism, misconduct, co-authorship, double sub-mission, etc.) have been considered carefully.

\section{Ethical Considerations}

\section{Compliance With Ethical Guideline}

In this research, all ethical considerations such as informed consent, preservation of anonymity and secrecy, voluntary participation in the interview were observed. The project was approved by research ethics committee of the University of Social Welfare and Rehabilitation Sciences.

\section{Funding}

This paper was extracted from a $\mathrm{PhD}$ dissertation. It did not receive any specific grant from funding agencies in the public, commercial, or not-for-profit sectors.

\section{Conflict of Interest}

The authors declare no conflict of interest. 


\title{
روشهاى استفادهشه براى حفظ و ارتقاى سلامت روان در خانمهاى متأهل ايرانى: يك مطالعه كيفى
}

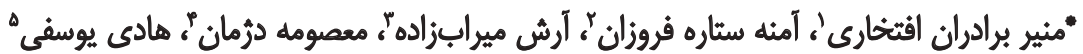

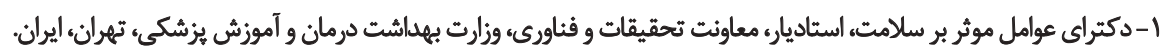

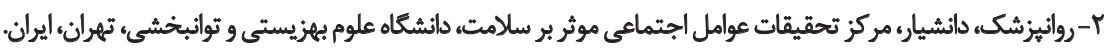

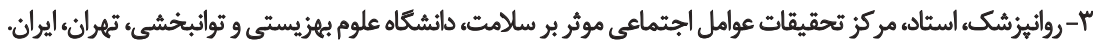

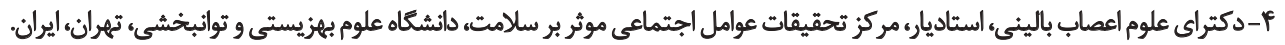

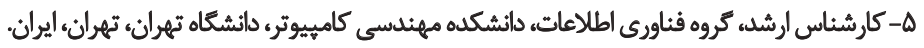

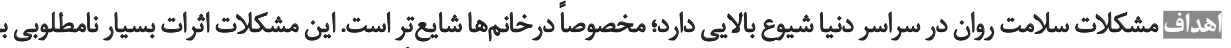

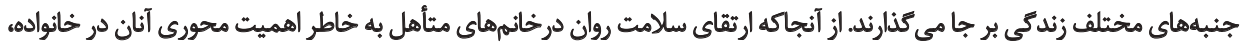

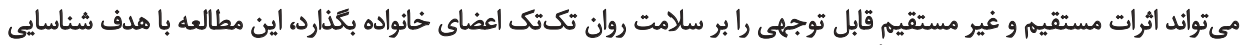

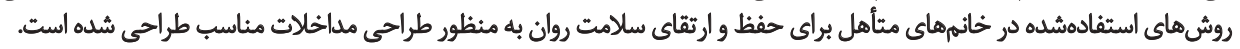

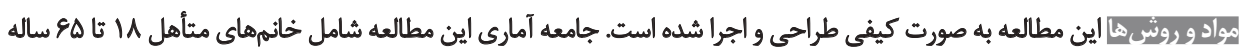

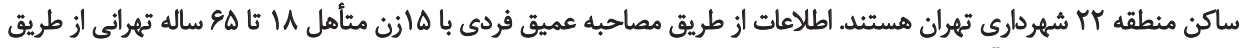

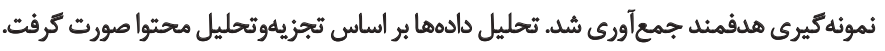

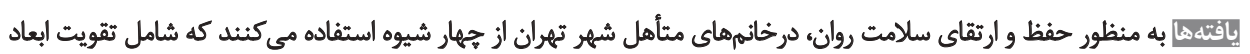

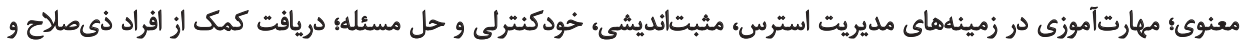

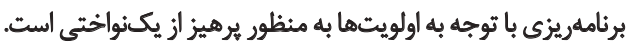

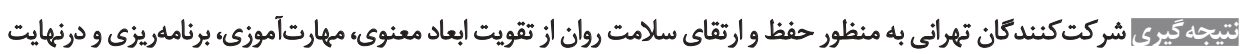

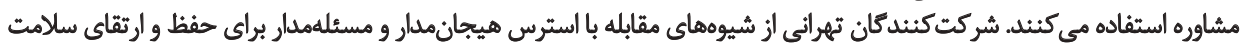
روان خود بيره مي كيرند.
تاريخ دريافت:

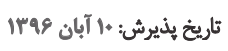

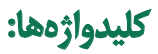
ارثقاى سلامت روان، زنان، مطالعه كيفى إمت رئري

$$
\begin{aligned}
& \text { هم اينك بيش از •هF ميليون نفر در سراسر دنيا از نوعى اختلال } \\
& \text { روانى رنج مىبرند [F] }
\end{aligned}
$$

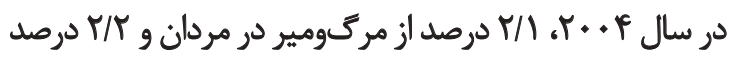

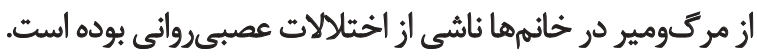

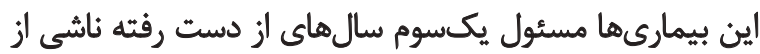

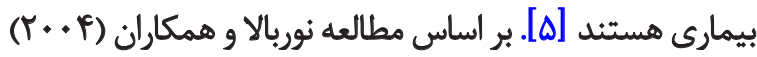

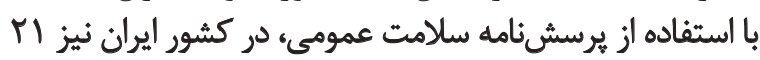

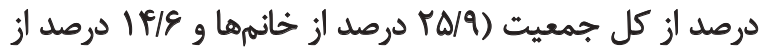

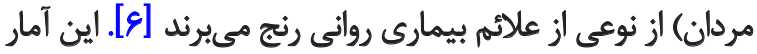

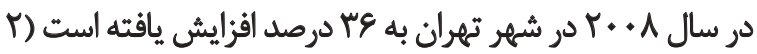

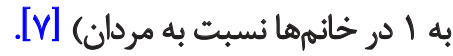
ارتقاى سلامت روان، در ساختار فرهنتى اجتماعى يك جامعه dale

سلامت روان حالتى از بهزيستى است كه افراد راقادر مي سازد تا

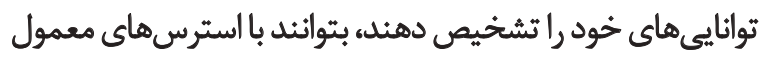

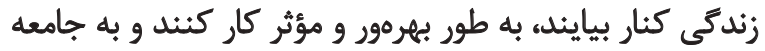

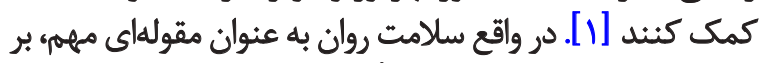

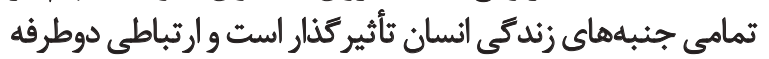

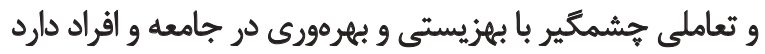

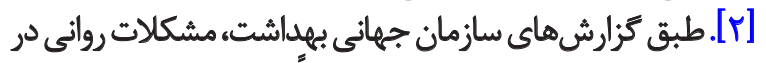

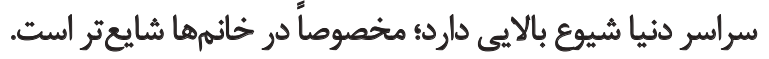

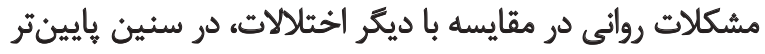

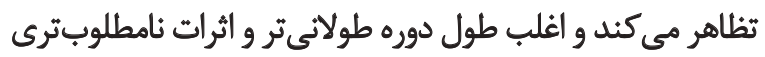

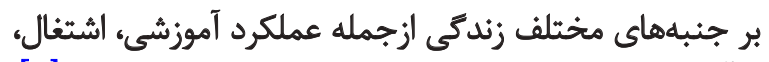

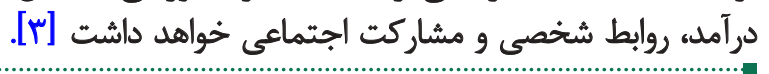

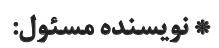
دكتر مثير برادران افتخارى مئن

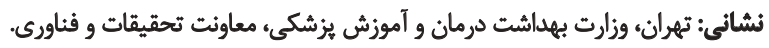

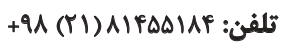
بيست الكترونيكي: mbeftekhari200@gmail.com 


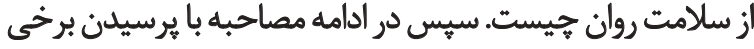

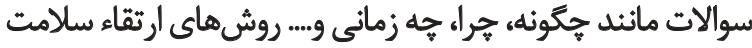

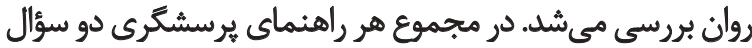

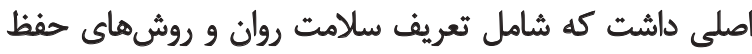

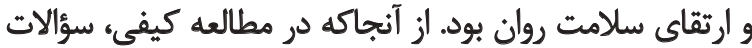

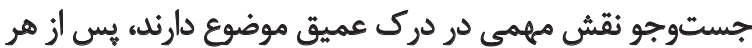

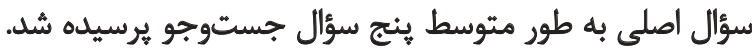

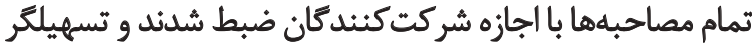

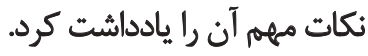

به منظور تجزيهوتحليل مصاحبهها از روش تحليل محتوا به

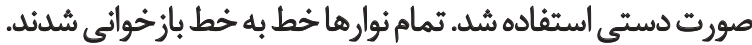

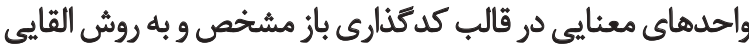

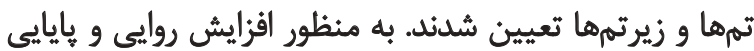

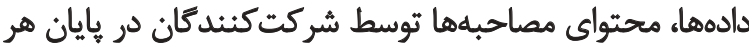

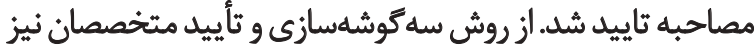

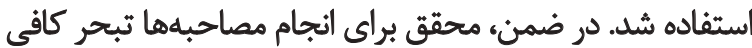

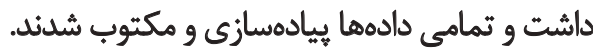

در اين يرؤهش تمام ملاحظات اخلاقى مانئد كسب رضايت

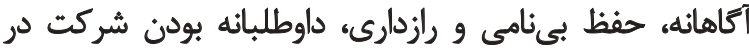

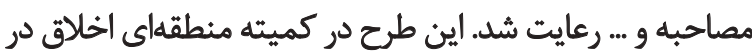

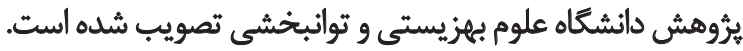

يافتهها

در اين مطالعه 10 مصاحبه عميق فردى صورت كرفت. ميانكين سن شركت كنيندكان • •

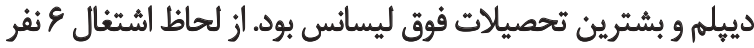

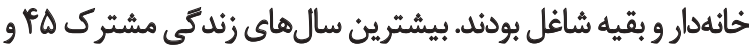

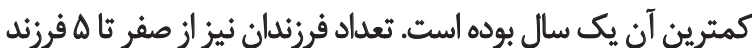

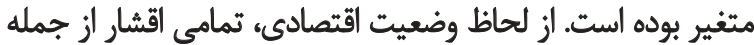

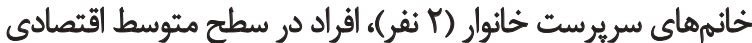

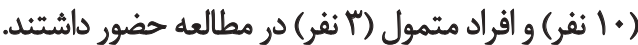

بر اساس تجزيلوتحليل انجامشده، جهار طبقه اصلى شامل

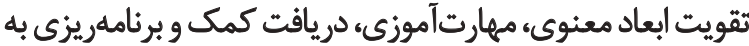

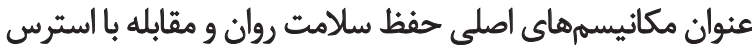

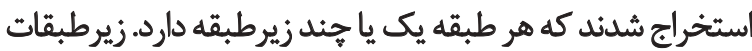

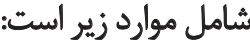

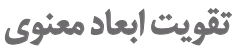

تمامى شركت كنندكان اعتقاد به خدا را به عنوان مكانيسم

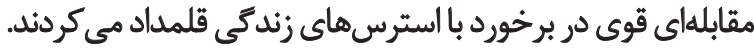

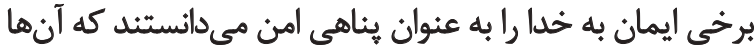
را در سختى ها يارى مى دهد. برخى نيز اعتقاد به خدا بندا را براى فرار

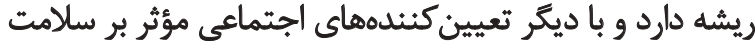

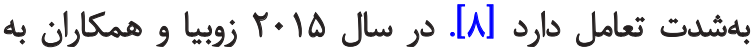

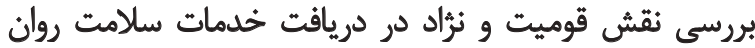

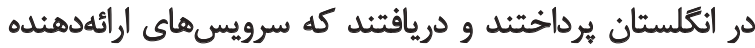

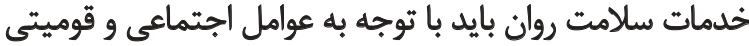

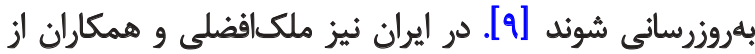

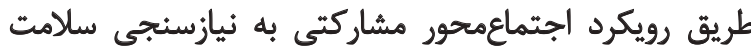

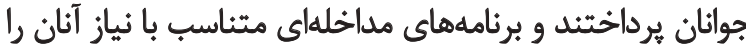

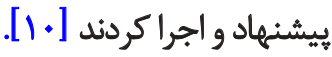

مطالعه حاضر، ارائه قسمتى از نتايج يك مطالعه كسترده

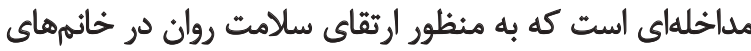

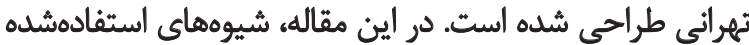

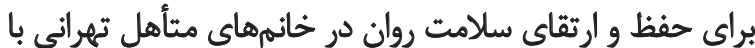
شيوه كيفى بررسي شفي ارته است

در اين مطالعه از روش تحقيق كيفى استفاده شده است اين است إني

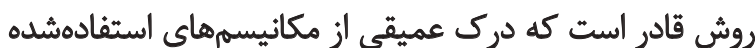

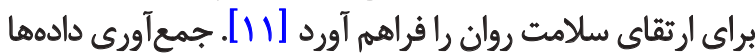

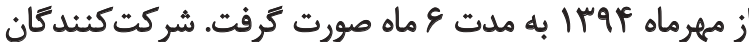

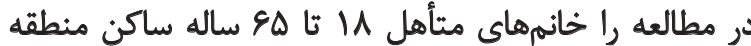

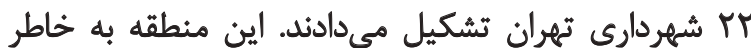

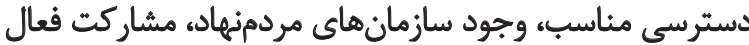

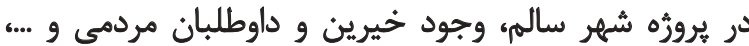

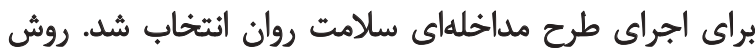

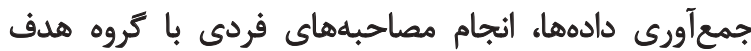

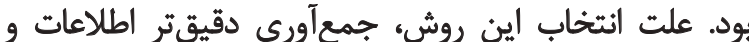

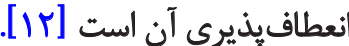

به منظور انجام مصاحبه، ابتدا از يك داوطلب كه شرايط ورود

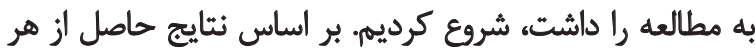

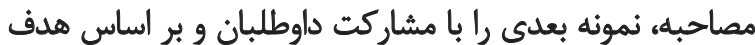

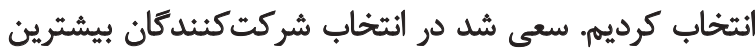

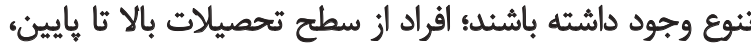

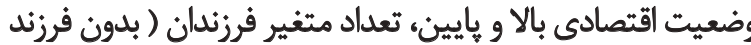

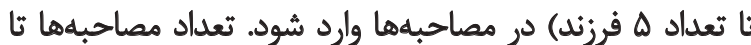

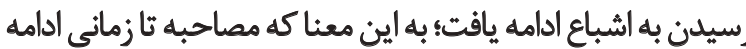

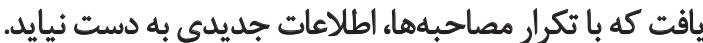
در اين مطالعه ها امصاحبه عميق فردى انجام شد. هر مصاحبه

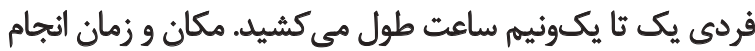

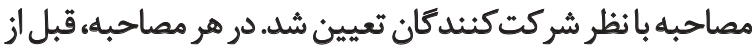

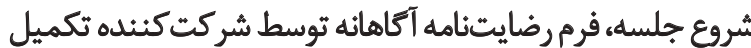

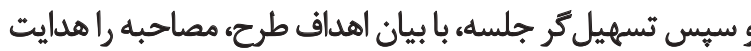
مى كرد. مصاحبه با اين سؤال كلى شروع مى شد كه تعريف شماف 
المن وقتى كه مشكلى برام بيش مياد، سعى مي كنهم به

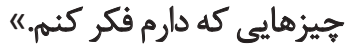

البه نظر من بايد هميشه نيمه بر ليوان را ديد؛ اين طورى آدم

$$
\text { خودكتيترلى راحتره }
$$

به نظر بسيارى از شركت كنندكان، تقويت اراده و اعتماد

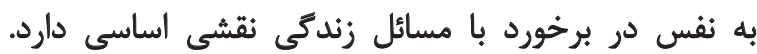

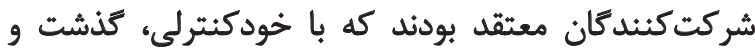

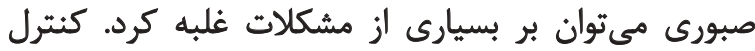

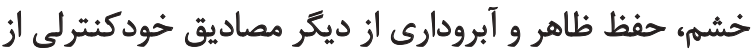

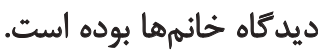

"اوقتى موقعيتى ييش مياد كه عصبانى ميشمه، سعى مي كنم بر خودم مسلط بشمر و خشمهم رو كنترل كنم."

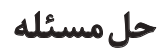

يكى از مهارت هاى مهمد بر برخورد باسترس هاسى روزمره، مهارت

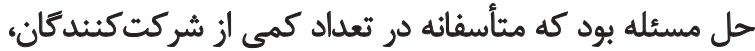

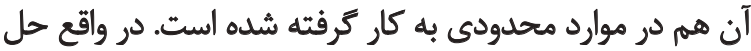

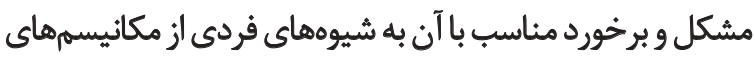

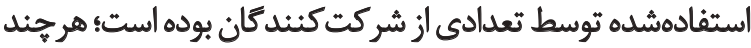
مراحل علمى و آكادميك آن را نمى آنسانستند.

وريافث كمك

برخى از خانمها معتقد بودند كه كُّشت زمان بسيارى از ازئ

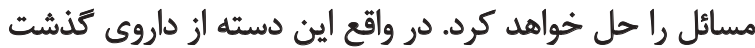

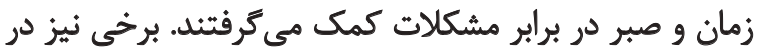

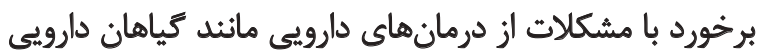

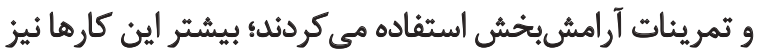

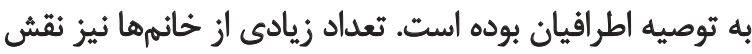

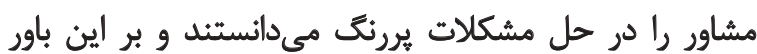

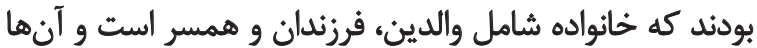

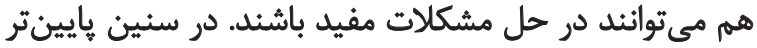

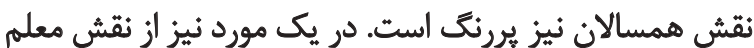
و يا استاد در حل مشكل ياد شد.

الامن هر وقت مشكلى برام بيش مياد، به شوهرم مى گمم. انغار وقتى باهاش حرف مىزنم، آروم مى بشمه "

الامن مىدونم كه رفتن بهرلوى مشاور خوبه، ولى جيكار كنم

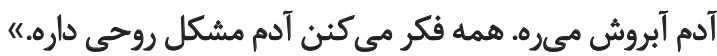

"امن هر وقت مشكلى برام ييش مياد، بادوستام حرف مىزنم."

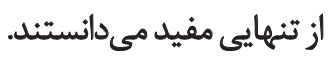

المن هر وقت احساس تنهايى مى كنم و كارى ازم برنمياد،

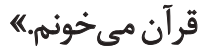

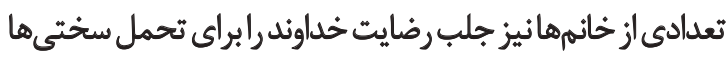

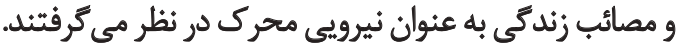

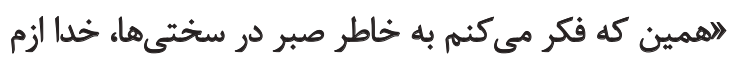

$$
\text { راضيه، انرزى مى كيرم. }
$$

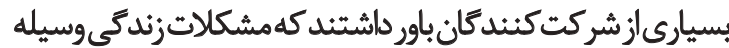

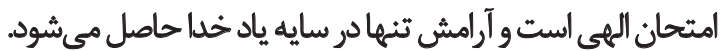

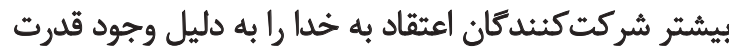

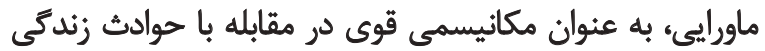

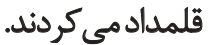

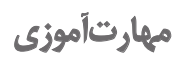

شركت كنئدكان بر اين عقيده بودند كه در برخورد با استرسها

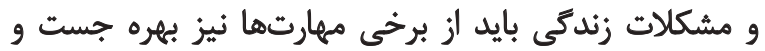

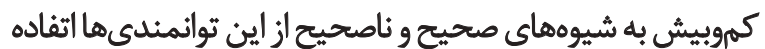

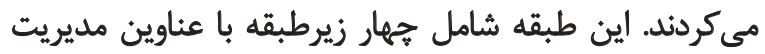

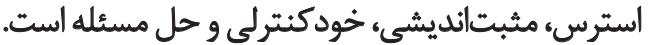

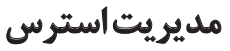

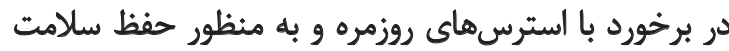

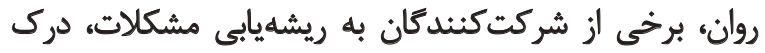

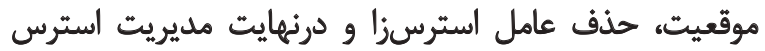

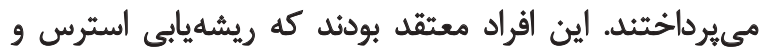

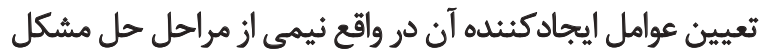

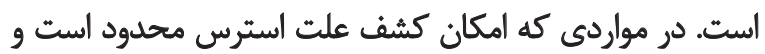

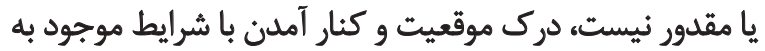

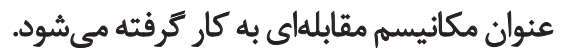

"اوقتى كارى نمى تونم بكنمه بهتره با خودم كنار بيام و

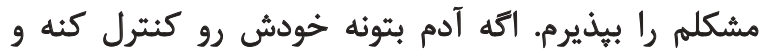

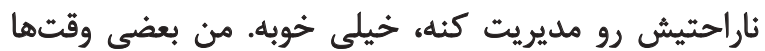
مي تونم اين كار رو بكنه.،

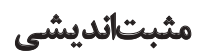
يكى ديكر از مكانيسمهاى ارتقاى سلامت روان، مثبتانديشى

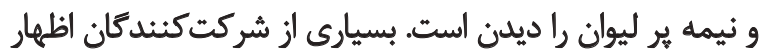

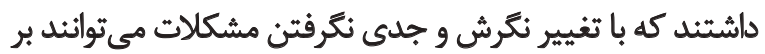

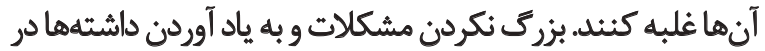

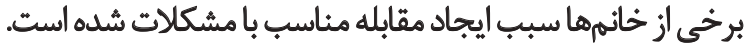


در مطالعه حاضر نيز تعابيرى كه توسط شركت كنيدكان در

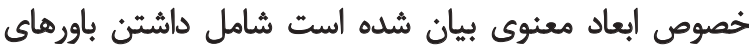

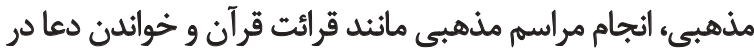

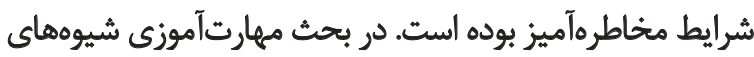

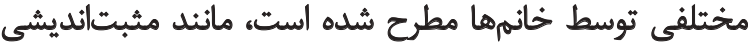

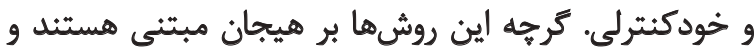

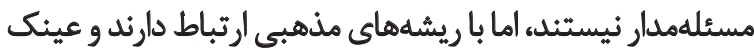

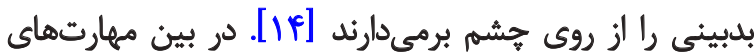

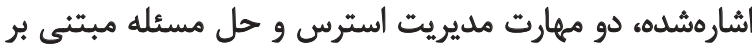

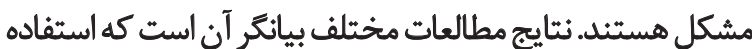

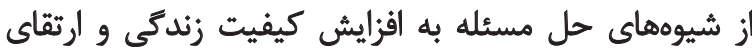

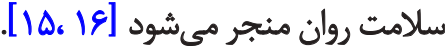
درخواست كمك نيز از جمله كنش/ كنش متقابل است كه

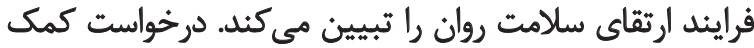

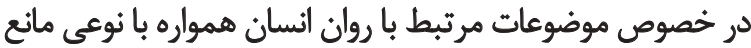

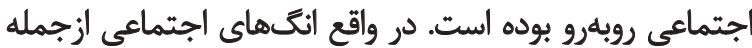

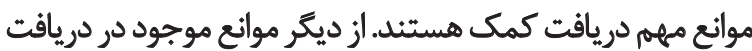

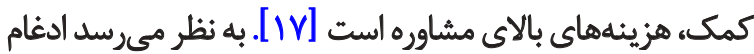

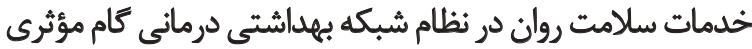
براى رفع موانع درخواست كمك است.

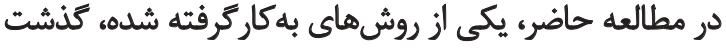

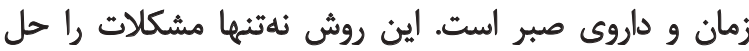

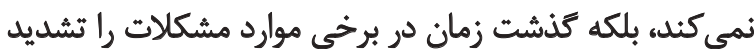

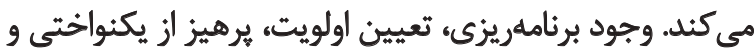

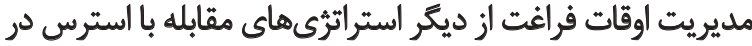

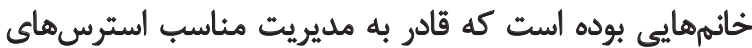

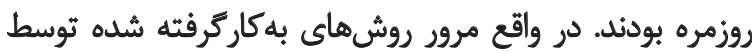

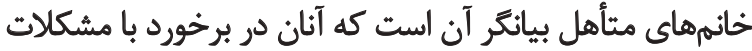

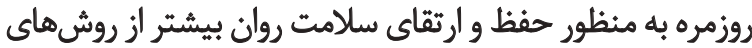

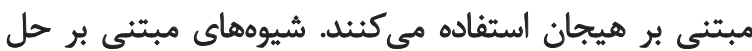

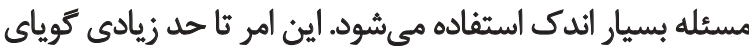
مشكلات متعدد آنان در برخورد با مسائل و مشكلات اتدات روزمره

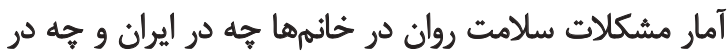

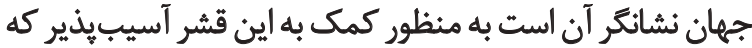

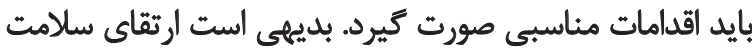

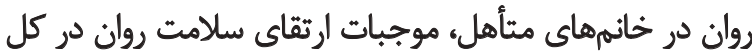

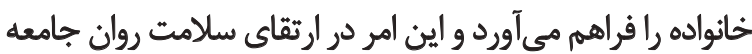

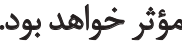

\section{نتيجليَّيرى}

به نظر مىرسد استفاده از روشهاى تحقيق كيفى به منظور درك عميق نيازهاى سلامت روان مى تواند سياست كذاران ان را در

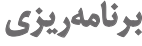

تعداد محدودى از شركت كنندكان كه بيشترشان تحصيل كرده

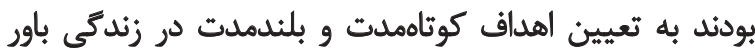

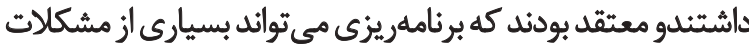

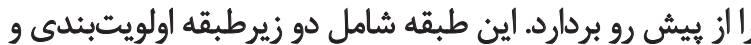
يرهيز از يكنواحتى است. برائ.

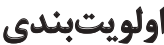

برخى از شركت كنيدكان بر اين باور بودند كه تعيين اولويتهاى

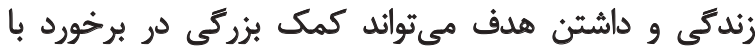

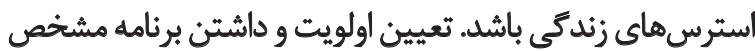

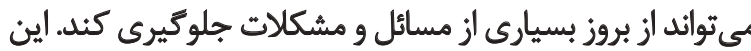
امر بيشتر در مسائل اقتصادى كاربرد مارد دارد. الوقتى براى زندكيم برنامه داشته باشم، طورى خرج مى كنم

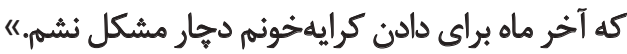

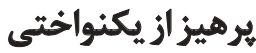

بسيارى از شركت كنثدكان معتقد بودند كه وجود يكنواختى

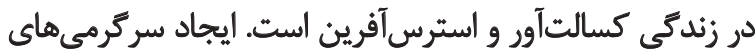

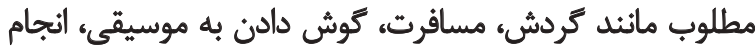

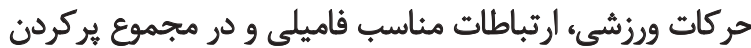

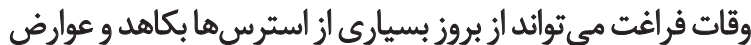

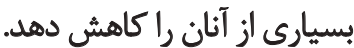

"المن وقتى دجار استرس مىشمه موسيقى توش مىدم،

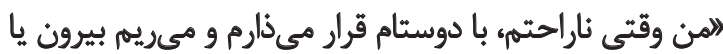

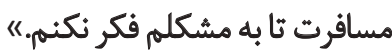

با مرور نتايج حاصل از اين مطالعه درمىيابيم كه خانمها براي

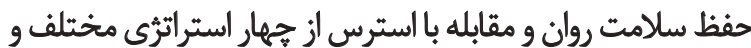

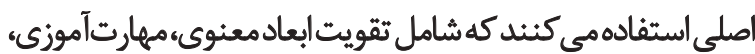

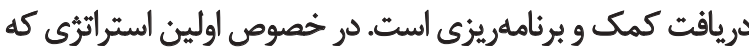

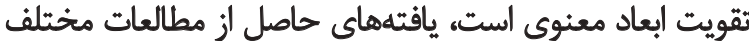

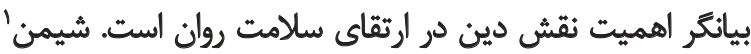

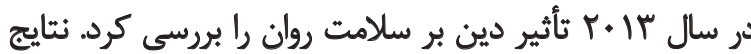

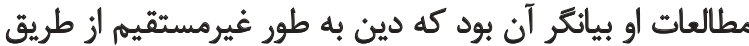

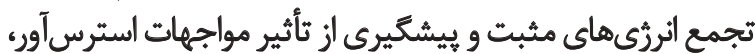

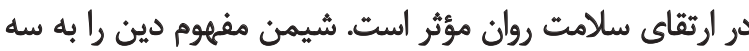

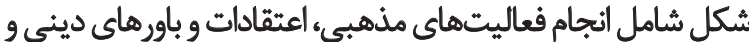

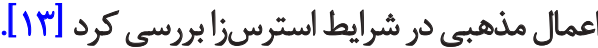


تدوين برنامههاى مداخلهاى مؤثرتر، يارى كند. با توجه به انجام

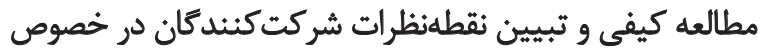

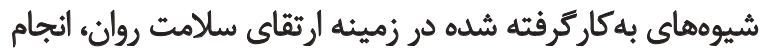

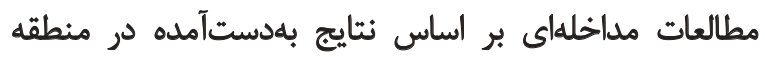

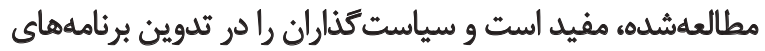

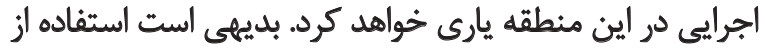

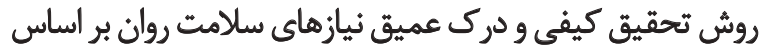

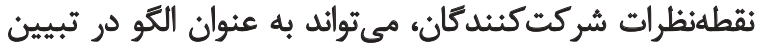
برنامههاى ارتقاى سلامت روان مؤثر باشد.

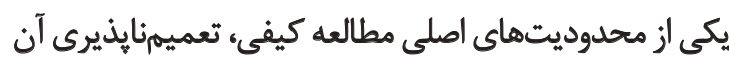

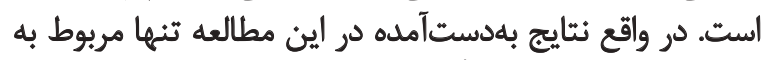

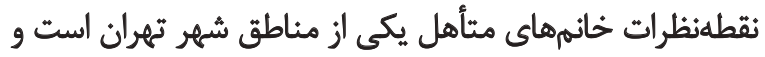
نتايج آن در نقاط ديكر قابل تعميم نيست ينان

$$
\text { سياسكّرَارى }
$$

اين مقاله بخشى از رساله دوره دكتراى تخصصى بوده و حامى مالى در سطح دانشكاه نداشته است. 


\section{References}

[1] World Health Organization. Mental health atlas 2005. Geneva: World Health Organization; 2005.

[2] Cattan M, Tilford S. Mental health promotion: A lifespan approach. New York: McGraw-Hill International; 2006.

[3] World Health Organization. Mental health: Strengthening our response (Fact sheet No. 220). Geneva: World Health Organization; 2010.

[4] Burgermeister D, Kwasky A, Groh C. Promoting mental health concepts in a doctor of nursing practice curriculum: An integrated and global approach. Nurse Education in Practice. 2012; 12(3):148-52. doi: 10.1016/j.nepr.2011.10.012

[5] Hughes JR. Nicotine dependence and WHO mental health surveys. JAMA. 2004; 292(9):1021-2. doi: 10.1001/jama.292.9.1021-c

[6] Noorbala AA, Yazdi SAB, Yasamy MT, Mohammad K. Mental health survey of the adult population in Iran. British Journal of Psychiatry. 2004; 184(1):70-3. doi: 10.1192/bjp.184.1.70

[7] Sarr PT, Kasturiarachchi CJ, Yang H, Co CJ, Shimpo A, Fujino $\mathrm{S}$, et al. Investigating the motivating factors behind high delivery rates of the Urban HEART birthing facility in San Martin de Porres, Philippines. Journal of the National Institute of Public Health. 2010; 59(1):64-70.

[8] Fisher M, Baum F. The social determinants of mental health: Implications for research and health promotion. Australian \& New Zealand Journal of Psychiatry. 2010; 44(12):1057-63. doi: 10.3109/00048674.2010.509311

[9] Islam Z, Rabiee F, Singh SP. Black and minority ethnic groups' perception and experience of early intervention in psychosis services in the United Kingdom. Journal of Cross-Cultural Psychology. 2015; 46(5):737-53. doi: 10.1177/0022022115575737

[10] Djalalinia S, Tehrani FR, Afzali HM, Peykari N, Eftekhari MB. Community mobilization for youth health promotion: A lesson learned from Iran. Iranian Journal of Public Health. 2012; 41(4):5562.

[11] Oun MA, Bach C. Qualitative research method summary. Journal of Multidisciplinary Engineering Science and Technology. 2014; 1(5):252-8.

[12] Guion LA, Diehl DC, McDonald D. Conducting an in-depth interview. Florida: University of Florida; 2001.

[13] Schieman S, Bierman A, Ellison CG. Religion and mental health In: Scheid T, editor. Handbook of the sociology of mental health. Amsterdam: Springer; 2012. doi: 10.1007/978-94-007-4276-5_22

[14] Prasa BA. Stress and coping parents with child mental retardation. Empathy. 2012; 1(1):1-10.

[15] Farhady A, Nooralizadeh M. The Effect of life-skill training on life satisfaction. Paper presented at The $2^{\text {nd }}$ International and $6^{\text {th }}$ Iranian National Conference on Health Education and Promotion; 19-21 May 2015; Kermanshah, Iran.

[16] Moshki M, Atarodi BA, Moslem A, Taheri M. Applying an educational-participatory program based on the PRECEDE model for promoting self-esteem and mental health of students in Iran. International Journal of Preventive Medicine. 2012; 3(4):241. PMCID: PMC3354393
[17] Rüsch N, Angermeyer MC, Corrigan PW. Mental illness stigma: Concepts, consequences, and initiatives to reduce stigma European Psychiatry. 2005; 20(8):529-39. doi: 10.1016/j.eurpsy.2005.04.004 
